\title{
Exhaustive catalytic ortho-alkoxylation of azobenzenes: flexible access to functionally diverse yellow-light-responsive photoswitches
}

\author{
Adrian Müller-Deku' ${ }^{1}$, Oliver Thorn-Seshold ${ }^{1, *}$ \\ 1: Department of Pharmacy, Ludwig-Maximilians University, Butenandtstrasse 5-13, Munich 81377, Germany. \\ ORCIDs: A.M-D. 0000-0001-6050-2545; O.T.-S. 0000-0003-3981-651X \\ * Correspondence to O.T.-S. (oliver.thorn-seshold@cup.lmu.de)
}

Keywords: azobenzene, C-H activation, photoswitch, photopharmacology, catalysis.

\begin{abstract}
We develop the first method for catalytic, exhaustive ortho-alkoxylation of azobenzene photoswitches. Alkoxylation is known to improve the photoswitch properties that control azobenzenes' success in chemical biology or materials sciences: e.g. better completeness of both $E \rightarrow Z$ and $Z \rightarrow E$ photoisomerisations, and $>100 \mathrm{~nm}$ red-shifting of photoresponse. Our method enables straightforward late-stage diversification of photoswitches with interesting functional handles. We showcase four applications, using it to rationally tune lipophilicity, prepare isotopic tracers for metabolism studies, install full water solubility without ionic charges, and efficiently access previously difficult mixed-substituent photoswitches. We also identified a previously unstudied mixed-substituent tetra-ortho-family, difluoro-dialkoxy-azobenzenes, whose photoresponse can outperform previous 'gold standard' tetrafluoro-, dichloro-difluoro-, and tetrachloro-azobenzenes in significant ways. We thus expect that both the scaffolds we showcase and the method we develop will impact broadly on photochemistry and photopharmacology.
\end{abstract}

\section{INTRODUCTION}

Reversible $E \rightarrow Z$ and $Z \rightarrow E$ isomerisations of azobenzenes can be effected with UV/visible light ${ }^{1}$, as well as with redox reactions following photocatalysis ${ }^{2}$, electrocatalysis ${ }^{3}$, or X-ray illumination ${ }^{4}$. The high spatiotemporal precision, non-invasiveness, and rapidity with which bulk populations of azobenzenes can be photoisomerised in both $E \rightarrow Z$ and $Z \rightarrow E$ directions has made azobenzene photoswitching a powerful tool for manipulating the physical and optical properties of solid materials ${ }^{5}$, the electronic and dynamic properties of soft matter $^{6}$, and for controlling protein functions and downstream cascades in biochemistry, cell biology, and in adult animals. ${ }^{7-12}$

Azobenzene photoresponse and isomerisation completeness are critical for all these applications. Alkylazobenzenes respond efficiently to light only up to ca. $500 \mathrm{~nm}$. They are often best photoisomerised around $360 \mathrm{~nm}$ (giving photostationary states (PSSs) of ca. 20:80 E:Z), and around $450 \mathrm{~nm}$ (PSSs ca. 80:20 E:Z). ${ }^{13,14}$

Several azobenzene substitution patterns have been developed to tune the isomers' spectra, and thus the photoresponsiveness and completion of photoisomerisations. ${ }^{15}$ Tetra-ortho-substitutions particularly improve photoswitch performance, without necessarily compromising function (as most azobenzene photopharmaceuticals are "azo-extension" or "azo-linker" designs ${ }^{16}$ where only the 4,4' positions determine bioactivity). ${ }^{17-20}$ Hecht's tetra-ortho-fluoro pattern gives excellent $E \rightarrow Z$ isomerisation at $500 \mathrm{~nm}$ and $Z \rightarrow E$ at $400 \mathrm{~nm}^{21}$ Woolley's sterically congested tetra-ortho-methoxy, ${ }^{22}$-thioalkyl ${ }^{23}$, and -chloro ${ }^{24}$ azobenzenes improved on older tetra-ortho-alkyl azobenzenes, ${ }^{25,26}$ with still better $E \rightarrow Z$ isomerisation at $550 \mathrm{~nm}$ (for good biocompatibility and depth penetration) making them alluring for photopharmacology in vivo; ${ }^{12} Z \rightarrow E$ isomerisation is most complete at $400 \mathrm{~nm}$ (see Supporting Note 1). ${ }^{21,22}$ However, most tetra-ortho syntheses are inflexible, needing ortho-substituents to be introduced early and carried through synthesis; ${ }^{24,27}$ the only exhaustive late-stage ortho-derivatisation reported is tetra-ortho-chlorination (Fig 1a). ${ }^{20}$

a

Trauner, 2016: late-stage $\mathrm{C}-\mathrm{H}$ tetrachlorination

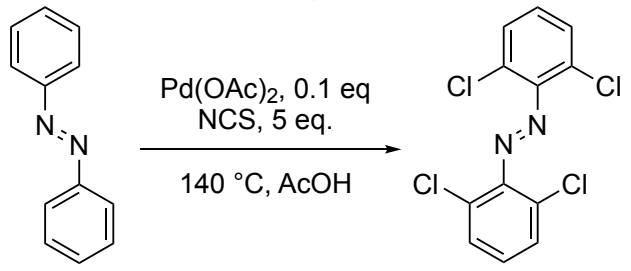

b Sun, 2013: azo-directed C-H monoalkoxylation

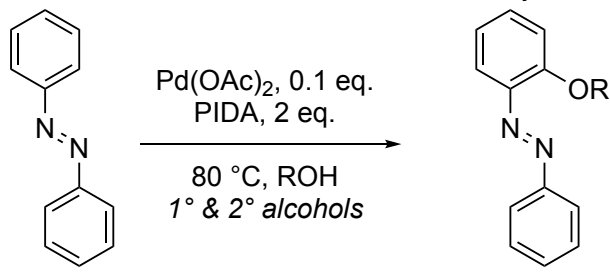

c

This work: late-stage $\mathrm{C}-\mathrm{H}$ tetraalkoxylation

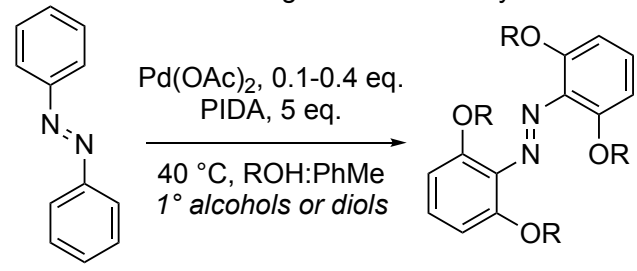

Figure 1. Towards exhaustive ortho-alkoxylation. (a) Trauner's Pd-catalysed per-ortho-chlorination. ${ }^{20}$ (b) Sun's Pd-catalysed mono-orthoalkoxylation. ${ }^{28}$ (c) Pd-catalysed per-ortho-alkoxylation reported here. 
The particular allure of the tetra-ortho-alkoxy pattern is that, unlike tetra-halides, it offers four ideal sites for functional diversification (as the alkyl part should not affect photoresponse). Yet, lacking a modular tetra-ortho-alkoxylation method, essentially no scope of functional ortho-substituents has been explored: e.g. these positions have never been exploited for solubilisation, isotopic labelling, or rational $\log \mathrm{D}$ tuning. Continuing our interest in polyalkoxylated photopharmaceuticals, ${ }^{9,29,30}$ but aiming to avoid polyphenol alkylations (see Supporting Note 1), we here aimed to develop a flexible, latestage, exhaustive ortho-alkoxylation procedure for azobenzenes.<smiles>N=Nc1ccccc1</smiles>
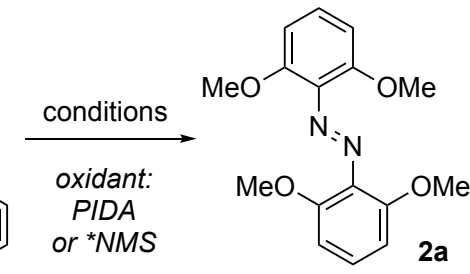

\begin{tabular}{ccccc} 
entry & $\mathrm{T}\left[{ }^{\circ} \mathrm{C}\right.$ ] & solvent / OMe eq. & $\mathrm{Pd}(\mathrm{OAc})_{2}$ [eq.] & isol. yield [\%] \\
\hline $\mathbf{1}$ & 140 & $\mathrm{AcOH} / \mathrm{NMS}^{*}$ & 0.10 & - \\
$\mathbf{2}$ & 80 & $\mathrm{MeOH}$ & 0.10 & $\mathbf{6}$ \\
$\mathbf{3}$ & 60 & $\mathrm{MeOH}$ & 0.10 & $\mathbf{9}$ \\
$\mathbf{4}$ & 40 & $\mathrm{MeOH}$ & 0.10 & $\mathbf{2 1}$ \\
$\mathbf{5}$ & 40 & $\mathrm{MeOH}: \mathrm{PhMe} \mathrm{1:1}$ & 0.10 & $\mathbf{2 8}$ \\
$\mathbf{6}$ & 40 & $\mathrm{MeOH}:$ PhMe 1:1 & 0.20 & $\mathbf{3 3}$ \\
$\mathbf{7}$ & 40 & MeOH:PhMe 1:1 & 0.40 & $\mathbf{3 8}$
\end{tabular}

Figure 2. Initial optimisation for temperature, cosolvent, and catalyst loading [NMS: $N$-methoxysuccinimide].

\section{Results}

\section{Catalytic tetra-ortho-methoxylation of azobenzene}

Palladium-catalysed $\mathrm{CH}$-oxidation using the diazene as an orthodirecting group was developed from a stoichiometric into a catalytic, exhaustive ortho-chlorination method by Trauner, ${ }^{20}$ with $\mathrm{N}$ chlorosuccinimide (NCS) acting as both oxidant and chlorine source (Fig 1a). We first tested if alkoxy analogues of NCS, e.g. $\mathrm{N}$ methoxysuccinimide [NMS], could likewise function as both oxidant and alkoxy source, but saw no conversion (Fig2, entry 1).

We then moved to alcohols as straightforward alkoxy sources, seeking tandem oxidation by another reagent to complete the turnover. As far as we know, Sun performed the only study of oxidative azobenzene ortho-alkoxylation, isolating mono-ortho-alkoxyazobenzenes in up to $77 \%$ yield by reacting a variety of non-, mono-meta-, and bis-meta-substituted azobenzenes with $\mathrm{Pd}(\mathrm{OAc})_{2}(10 \mathrm{~mol} \%)$, alcohol, and $\mathrm{PhI}(\mathrm{OAc})_{2}$ [PIDA] oxidant (1-2 eq.) at $80^{\circ} \mathrm{C}$ (Fig 1b, mechanism in Scheme S2). ${ }^{28}$ However, despite superstoichiometric oxidant, no polyalkoxylation was reported. Clean oxidative peralkoxylation is anyway a challenging prospect, as each alkoxylation step will increase the substrate's electron density, so raising the chances of non-regiospecific or undesired oxidations (Scheme S2).

Aiming to achieve up to four $\mathrm{CH}$-oxidations per azobenzene for clean per-ortho-alkoxylation (Fig 1c), we began our quest using Sun's mono-methoxylation conditions but increasing oxidant to 5 mol. eq. (Table S1). HPLC-MS analysis revealed a low but encouraging 6\% yield (Fig 2, entry 2 ). Residual substrate and monomethoxy intermediate were the major impurities isolated, with di- and trimethoxylated intermediates as trace products only, matching the expectation of increasing reactivity per alkoxylation step.
However, we observed rapid formation of palladium black during heating, as expected for reduction of $\mathrm{Pd}(\mathrm{OAc})_{2}$ by methanol, ${ }^{31}$ likely blocking reaction progress. As reduction was slower at lower temperatures, we screened down to $40^{\circ} \mathrm{C}$ under strict temperature control, increasing the tetra-substitution yield to $28 \%$ (Fig 2, entries 34). This is unusual for $\mathrm{CH}$ activations, that are generally favoured by high temperatures. As nitrogen ligands can stabilise $\mathrm{Pd}(\mathrm{OAc})_{2}$ against reduction, we tested pyridine and 1,1'-bipyridine; while these prevented formation of palladium black, they also blocked alkoxylation (Table S1, entries 8-10).

\section{Cosolvent, oxidant, additives, and catalyst loading}

We next screened cosolvents to methanol (Table S1, entries 1125); toluene improved yields, with good results at 1:1 MeOH:PhMe (Fig 2, entry 5). We also screened diluted conditions, since the solubility of PIDA is only moderate: but these lowered isolated yields greatly (Table S1, entry 26), coherent with the role of a dimeric intermediate (Scheme S2). PIDA was however superior to PIFA, oxone, or $\mathrm{K}_{2} \mathrm{~S}_{2} \mathrm{O}_{8}$ as the oxidant, and increasing its loading (10 eq.) only reduced yields (Table S1, entries 27-32). This is coherent with expectations that excess or stronger oxidants drive parasitic oxidations of the increasingly electron-rich intermediates/product. Acidic, basic, or dehydrating additives also did not give improvements (Table S1, entries 33-40). Instead, reaction yields improved when $\mathrm{Pd}(\mathrm{OAc})_{2}$ loading was raised (Fig 2, entries 6-7).

Taken together, this screening had improved isolated yields of tetra-ortho-methoxylation to reach nearly $40 \%$ (Fig 2, entry 7), corresponding to ca. $75 \%$ yield per $\mathrm{CH}$ activation/C-O formation, which matches pleasingly to the single-step yields of Sun. ${ }^{28}$

\section{Scope for azobenzene substituents}

To study this method's substrate preference, we applied the conditions optimised for unsubstituted 1a to a set of mono-substituted azobenzenes (Fig 3). Electron-donating groups in para gave poor (alkyl 2b) to negligible (oxyether) yields, but were better tolerated in meta (2c-2d) (discussion at Scheme S2). Electron-poor parasubstituents were successful (ester 2e, halogens $\mathbf{2 f - 2 g}$, nitro $\mathbf{2 h}$ ). These patterns, which offer flexible derivatisation by reduction/acylation (nitro), esterification/amidation (ester), or cross-coupling (halide), can now be accessed by an easier and more tolerant route than previously possible with e.g. lithium base ${ }^{27}$ methods.

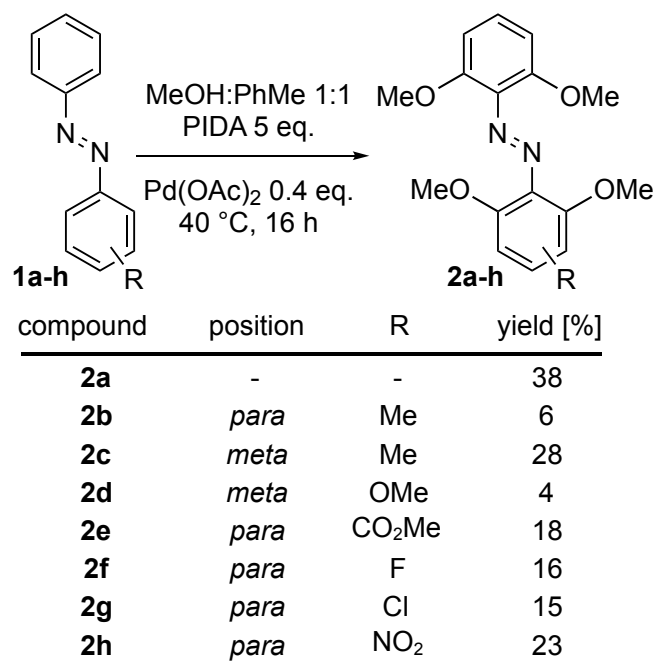

Figure 3. Substituent effects on tetra-methoxylation [isolated yields]. 


\section{ortho-alkoxylation as a functional handle}

To explore the scope for exhaustive alkoxylation to products other than tetramethoxy species, we tested alcohols other than methanol, and performed reactions on partially-ortho-substituted substrates (Fig 4). Secondary alcohols ( $i \mathrm{PrOH})$ gave only traces of tetraalkoxylation, and tertiary alcohols $(t \mathrm{BuOH})$ gave no conversion, which is understandable due to sterics. Though electron-poor primary alcohols $\left(\mathrm{CF}_{3} \mathrm{CH}_{2} \mathrm{OH}\right)$ were unreactive, we found good scope for primary alcohols, which enables tackling four novel applications:

(1) Lipophilicity is a key property for cellular pharmacology, impacting apparent on-target affinity, off-target binding, subcellular localisation, bioavailability, and membrane permeation. ${ }^{32}$ Late-stage per-alkoxylations could give diversified sets of spectrally identical photopharmaceuticals, with rationally tuned $\log \mathrm{D}$ values according to the alkoxy residues attached: for convenient validation of their mechanism of action, or for potency optimisation. Per-alkoxylation with OMe, OEt and OPr groups on mono-, di- and tri-alkoxy substrates (3a-3j) gave respectable yields (ca. 55\% per substitution).

\begin{tabular}{|c|c|c|c|c|c|}
\hline \multicolumn{2}{|c|}{$\begin{array}{l}\text { unsubstituted } \\
\text { or substituted } \\
\text { azobenzenes }\end{array}$} & \multicolumn{2}{|c|}{$\begin{array}{l}\text { ROH:PhMe } 1: 1 \\
\text { PIDA } 5 \text { eq. } \\
\underset{\mathrm{Pd}(\mathrm{OAc})_{2} 0.4 \text { eq. }}{4} \\
\quad 40^{\circ} \mathrm{C}, 16 \mathrm{~h}\end{array}$} & \multirow[b]{2}{*}{$\mathrm{R}^{4}$} & \multirow[b]{2}{*}{$\begin{array}{l}\text { yield [\% per } \\
\text { substitution] }\end{array}$} \\
\hline compound & $\mathrm{R}^{1}$ & $\mathrm{R}^{2}$ & $\mathrm{R}^{3}$ & & \\
\hline \multicolumn{6}{|c|}{ Lipophilicity Tuning } \\
\hline $3 a$ & OMe & OMe & OMe & $(\mathrm{OEt})$ & 54 \\
\hline $3 b$ & $(\mathrm{OMe})$ & OEt & $(\mathrm{OMe})$ & OEt & 40 \\
\hline $3 c$ & (OMe) & (OMe) & OEt & OEt & 67 \\
\hline $3 d$ & (OMe) & OEt & OEt & OEt & 54 \\
\hline $3 e$ & OEt & OEt & OEt & OEt & 58 \\
\hline $3 f$ & $(\mathrm{OMe})$ & $\mathrm{OPr}$ & $(\mathrm{OMe})$ & OPr & 53 \\
\hline $3 g$ & (OMe) & (OMe) & $\mathrm{OPr}$ & OPr & 88 \\
\hline $3 h$ & (OMe) & OPr & OPr & OPr & 63 \\
\hline $3 \mathbf{i}$ & $(\mathrm{OEt})$ & $\mathrm{OPr}$ & OPr & OPr & 43 \\
\hline $3 \mathbf{j}$ & OPr & OPr & OPr & OPr & 47 \\
\hline \multicolumn{6}{|c|}{ Mixed Substituents } \\
\hline $3 k$ & OMe & OMe & OMe & $(F)$ & 55 \\
\hline 31 & OMe & $(\mathrm{F})$ & OMe & $(\mathrm{F})$ & 33 \\
\hline $3 m$ & OMe & OMe & $(\mathrm{F})$ & $(F)$ & 22 \\
\hline \multicolumn{6}{|c|}{ Isotopic Labelling } \\
\hline $3 n$ & $\mathrm{OCD}_{3}$ & $\mathrm{OCD}_{3}$ & $\mathrm{OCD}_{3}$ & $\mathrm{OCD}_{3}$ & 70 \\
\hline \multicolumn{6}{|c|}{ Water Solubility } \\
\hline 30 & $\mathrm{OPrOH}$ & OPrOH & OPrOH & OPrOH & 45 \\
\hline
\end{tabular}

Figure 4. Per-ortho-alkoxylations of a range of azobenzenes giving otherwise difficult to access or unreported substituent patterns. Bracketed residues, e.g. $(\mathrm{OMe})$, were present in the starting materials; bold residues were introduced by the reaction [isolated yields].

(2) Asymmetry: The optical properties of symmetric tetra-orthosubstituted azobenzenes have been explored (see Introduction). However, only a few asymmetric tetra-ortho azobenzenes (where substituents differ from each other) have been studied, and these only recently: yet strong performance enhancements were already found. ${ }^{19}$ Exhaustive alkoxylation of partially ortho-substituted substrates offers a convenient synthetic access to otherwise time-intensive mixed-substituent patterns that have not yet been systematically explored. Pleasingly, mixed fluoro/alkoxy tetra-ortho azobenzenes 3k-3m were all accessed with moderate yields, for later study.

(3) Metabolism: As photopharmacology moves towards applications in adult animals, ${ }^{10-12}$ monitoring the metabolism of yellow/redlight responsive photoswitches will become increasingly urgent. We know of no reports preparing isotopically-labelled photopharmaceuticals for unbiased MS-based metabolite studies, which typically require light/heavy drug pairs with $>M+5$ for the heavy drug: $:^{33}$ but reasoned that exhaustive ortho-alkoxylation is an attractive method to do so. Using $\mathrm{CH}_{3} \mathrm{OH} / \mathrm{CD}_{3} \mathrm{OD}$ provides up to $\mathrm{M}+12$ difference in light/heavy masses of scaffold-intact metabolites, $\mathrm{M}+6$ difference of the principal hypothesised azobenzene metabolites $(\mathrm{N}=\mathrm{N}$ scission products), or M+9 difference of mono-ortho-demethylated species: all of which are sufficient for unbiased metabolite detection, and which may reveal the in vivo fate of photopharmaceuticals. Yields of tetra-ortho- $\left(\mathrm{OCD}_{3}\right)$ derivatisation were good (3n).

(4) Solubility: Like most photoswitches (typically, flat aromatics), azobenzenes have very poor water solubility and tend to aggregate; this complicates their photoswitching and spectra, ${ }^{13,34}$ and hampers applications from materials sciences through to biology. ${ }^{35}$ Exhaustive alkoxylation with diols could be a late-stage solubilising method that also provides beneficial tetra-ortho photoresponse, without crowding the para-positions needed for "azo-extension" and "azo-linker" photopharmacology, ${ }^{8}$ and without charges that complicate bioactivity. Tetra-alkoxylation with propylene glycol gave good isolated yields of water-soluble tetrol 30 ( $45 \%$ per substitution): the first example we know of such solubilisation in photoswitching.

\section{Photoswitching performance}

The tetra-ortho-alkoxyazobenzenes' optical properties matched Woolley's reports ${ }^{22,24}$. They absorb well in the visible, with substantial separation between the isomers' band maxima giving ca. $80 \% \mathrm{Z}$ at PSS under $550 \mathrm{~nm}$, and ca. 85\% $E$ at PSS under $405 \mathrm{~nm}$ (Fig 5ab, 3i/3o). Bulk photoswitching with blue and green light was efficient; and despite low absorption coefficients above $550 \mathrm{~nm}$ they can be isomerised by yellow light up to $600 \mathrm{~nm}$ (Fig 5c, Fig S2). The spontaneous $Z \rightarrow E$ relaxation of all derivatives was slow on relevant timescales (halflives $>7$ days; Fig S2a). Bidirectional photoswitching was fully reversible without detectable losses over tens of cycles (Fig S2b). Water-soluble tetrol 30 was assayed in all-aqueous buffer, supporting the utility of this approach for biology (Fig 5, Fig S2).

This straightforward access to per-ortho-alkoxylated azobenzenes can now drive systematic investigations, identifying promising new switches. For example, difluoro-dialkoxy-azobenzenes were only accessed once before, ${ }^{27}$ though their photoswitching was not investigated and they have not been practically applied. We saw that both asymmetric and symmetric difluoro-dialkoxy $\mathbf{3 m}$ and $\mathbf{3 l}$ had ca. $20 \mathrm{~nm}$ more separation between the $n \rightarrow \pi^{*}$ bands of their $E$ - and $Z$ isomers than tetra-alkoxy derivatives (Fig S2c). This greater separation drives excellent completeness of $E \rightarrow Z$ photoswitching under green light (only 4-5\% E remaining, Fig 5a), which outperforms tetra-ortho-alkoxy- (20\%) and even difluoro-dichloro-azobenzenes ${ }^{19}$ (15\%) by several fold, while all have comparable $Z \rightarrow E$ conversion. Symmetric 31 also has a ca. $20 \mathrm{~nm}$ red-shift of $E \rightarrow Z$ photoresponse compared to popular tetra-ortho-fluoro ${ }^{21}$ species (asymmetric $3 \mathbf{m}$ has a $30 \mathrm{~nm}$ blue-shift); and both enjoy the benefits of the flexible alkoxy handles. This performance and access recommend them for adoption, and should more broadly motivate studies of mixed substitution patterns to refine the photochemistry, biophysical properties, and functions accessible for photopharmacology. 
a

\begin{tabular}{|c|c|c|c|c|c|c|}
\hline $\begin{array}{l}\text { PSS } \\
\% Z\end{array}$ & $\overline{405}$ & 435 & 470 & 490 & 525 & 550 \\
\hline $3 \mathbf{i}$ & 11 & 16 & 43 & 55 & 78 & 81 \\
\hline $3 m$ & 18 & 23 & 66 & 90 & 94 & 95 \\
\hline 31 & 22 & 34 & 80 & 87 & 95 & 96 \\
\hline 30 & 16 & 18 & 44 & 57 & 76 & 80 \\
\hline
\end{tabular}

b

PSS Spectra
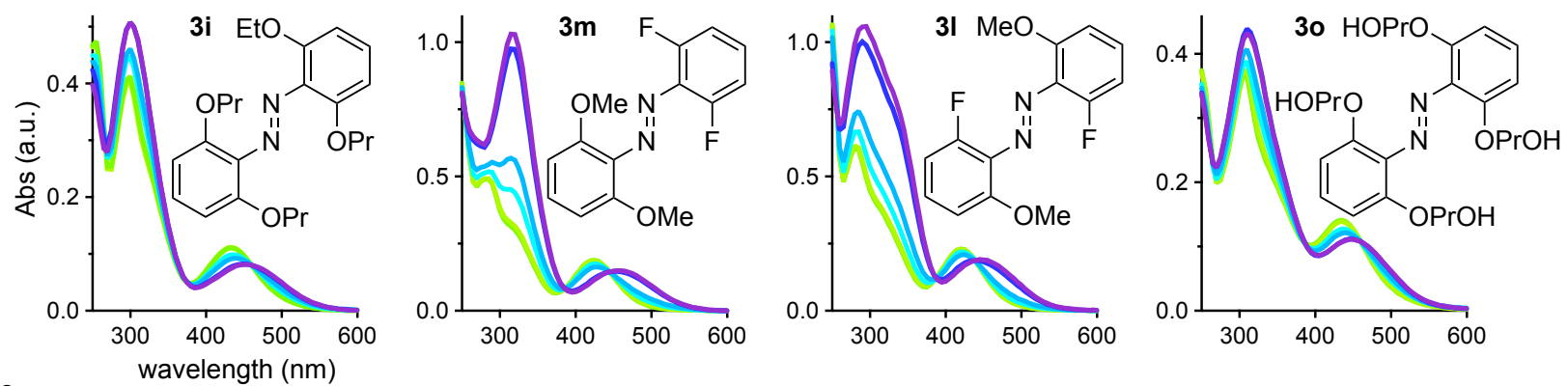

Photoresponse Kinetics
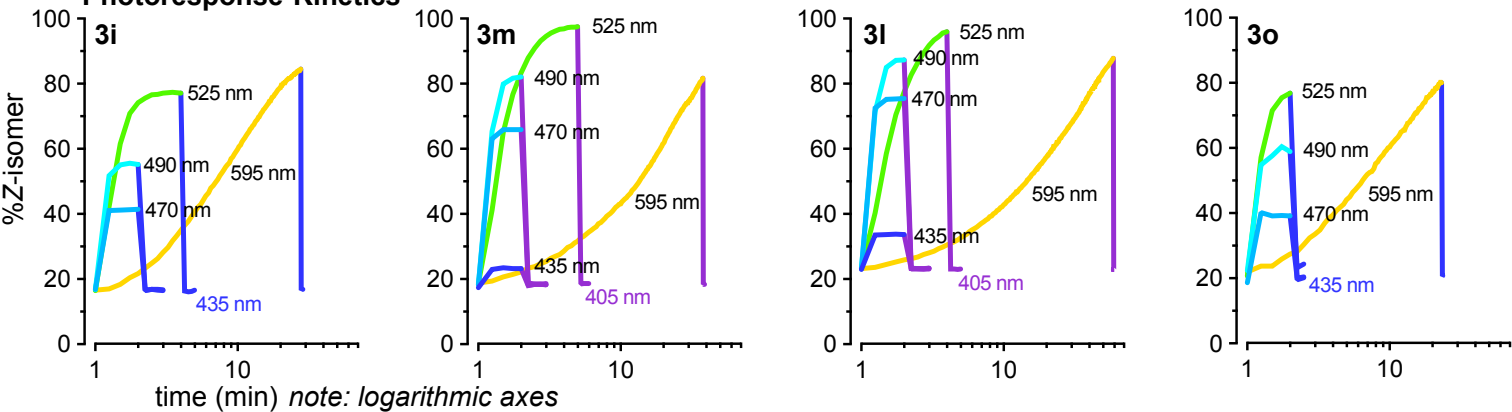

Figure 5. Optical properties. (a-b) Photostationary state (PSS) $E: Z$ proportions (by HPLC) and corresponding UV-Vis absorption spectra. (c) $E \rightleftharpoons Z$ photoisomerisation timecourses illustrating variations of photoresponse speed and completeness with wavelength. $E \rightarrow Z$ photoswitching was performed with the annotated test wavelengths starting at $\mathrm{t}=1 \mathrm{~min}$; at the end of each test, $Z \rightarrow E$ back-switching was performed at $435 \mathrm{~nm}$ or $405 \mathrm{~nm}$ to verify photoreversibility (LED sources, see discussion at Fig S2-S3). Spectra measured in MeCN (3i, 3m, 31) or in deionised water (3o).

\section{Conclusion}

Tetra-ortho-alkoxyazobenzenes feature all-visible-light bidirectional photoswitching that is substantially complete in both $E \rightarrow Z$ and $Z \rightarrow E$ directions. ${ }^{22}$ Unlike bridging ${ }^{36,37}$ or tetra-ortho-halogenation ${ }^{19,21}$ approaches to redshift azobenzene photoresponses, per-ortho-alkoxylation offers up to four flexible handles for installing functionality, potentially without compromising bioactivity or Z-isomer stability.

We here present the first method for exhaustive ortho-alkoxylation of electron-poor to electron-neutral azobenzenes with primary alcohols, giving isolated yields around $50-75 \%$ per alkoxylation. The congested structures accessible by this method are not easily accessible otherwise. It both enables late-stage diversification, and also simplifies synthesis in general by avoiding the need to install alkoxy substituents before azobenzene formation.

We also present method applications that may extensively impact photopharmacology (Fig 4): stepwise tuning of lipophilicity in a spectrally identical series of derivatives; poly-deuteration for unbiased metabolism studies; conversion to water-soluble uncharged polyols; and new access to hitherto poorly-studied switches. The latter led us to identify difluoro-dialkoxy-azobenzenes as highly performant photoswitches (Fig 5). Since the greater sensitivity of tetra-ortho-alkoxy-azobenzenes to bithiol-mediated degradation (as compared to their tetra-ortho-halo counterparts) is a concern for longterm biological studies, ${ }^{24}$ this method towards new difluoro-dialkoxy switches may prove particularly valuable for chemical biology: by finally combining the biological stability of halogenation with the functional flexibility of alkoxylation (on top of the general photophysical benefits of tetra-ortho-substitution patterns).

This straightforward and flexible method for functional photoswitch tuning can therefore promote access to a range of efficiently and bidirectionally visible-light responsive, metabolically traceable, solubilised, and rationally tunable azobenzene photoswitches, with particular applications to materials sciences and chemical biology.

\section{ASSOCIATED CONTENT}

\section{Supporting Information}

The Supporting Information is available free of charge on the ACS Publications website.

PDF containing synthetic protocols; photocharacterisation; and NMR spectra.

Datafile (XLSX) with $E / Z$ and PSS spectra of selected compounds.

\section{AUTHOR INFORMATION}

\section{Corresponding Author}

* Correspondence to O.T.-S. (oliver.thorn-seshold@cup.lmu.de)

\section{Author Contributions}

A.M.-D. performed all synthesis, characterisation, and data assembly. O.T.-S. designed the study, supervised experiments, and wrote the manuscript. 


\section{Funding Sources}

This research was supported by funds from the German Research Foundation (DFG: SFB1032 Nanoagents for Spatiotemporal Control project B09 number 201269156; SFB TRR 152 project P24 number 239283807, Emmy Noether grant TH2231/1-1 number 400324123, and SPP 1926 project number 426018126).

\section{ACKNOWLEDGMENT}

We thank Nurunnisa Özyürek and Nesrin Hamou (LMU) for synthesis, Dr. Lars Allmendinger (Zentral Analytik, LMU) for NMR, and Martin Maier (LMU) for manuscript feedback.

\section{ABBREVIATIONS}

NMS: $N$-methoxysuccinimide; PIDA, phenyliodine(III) diacetate; PIFA, phenyliodine(III) bis(trifluoroacetate; PBS, phosphate-buffered saline.

\section{REFERENCES}

(1) Jerca, F. A.; Jerca, V. V.; Hoogenboom, R. Advances and Opportunities in the Exciting World of Azobenzenes. Nat. Rev. Chem. 2021, 1-19. https://doi.org/10.1038/s41570-021-00334-w.

Goulet-Hanssens, A.; Rietze, C.; Titov, E.; Abdullahu, L.; Grubert, L.; Saalfrank, P.; Hecht, S. Hole Catalysis as a General Mechanism for Efficient and Wavelength-Independent $\mathrm{Z} \rightarrow \mathrm{E}$ Azobenzene Isomerization. Chem 2018, 4 (7), 1740-1755. https://doi.org/10.1016/j.chempr.2018.06.002.

(3) Greenfield, J. L.; Gerkman, M. A.; Gibson, R. S. L.; Han, G. G. D.; Fuchter, M. J. Efficient Electrocatalytic Switching of Azoheteroarenes in the Condensed Phases. J. Am. Chem. Soc. 2021, 143 (37), 15250-15257. https://doi.org/10.1021/jacs.1c06359.

(4) Ober, M. F.; Müller-Deku, A.; Baptist, A.; Amenitsch, H.; ThornSeshold, O.; Nickel, B. SAXS Measurements of Azobenzene Lipid Vesicles Reveal Buffer-Dependent Photoswitching and Quantitative Z->E Isomerisation by X-Rays. ArXiv211112570 Cond-Mat Physicsphysics 2021.

(5) Russew, M.-M.; Hecht, S. Photoswitches: From Molecules to Materials. Adv. Mater. 2010, 22 (31), 3348-3360. https://doi.org/10.1002/adma.200904102.

(6) Boelke, J.; Hecht, S. Designing Molecular Photoswitches for Soft Materials Applications. Adv. Opt. Mater. 2019, 7 (16), 1900404. https://doi.org/10.1002/adom.201900404.

(7) Beharry, A. A.; Woolley, G. A. Azobenzene Photoswitches for Biomolecules. Chem. Soc. Rev. 2011, 40 (8), 4422-4437. https://doi.org/10.1039/C1CS15023E.

(8) Hüll, K.; Morstein, J.; Trauner, D. In Vivo Photopharmacology. Chem. Rev. 2018, 118 (21), 10710-10747. https://doi.org/10.1021/acs.chemrev.8b00037.

(9) Borowiak, M.; Nahaboo, W.; Reynders, M.; Nekolla, K.; Jalinot, P.; Hasserodt, J.; Rehberg, M.; Delattre, M.; Zahler, S.; Vollmar, A.; Trauner, D.; Thorn-Seshold, O. Photoswitchable Inhibitors of Microtubule Dynamics Optically Control Mitosis and Cell Death. $\begin{array}{lllll}\text { Cell 2015, } & 162 & \text { (2), }\end{array}$ https://doi.org/10.1016/j.cell.2015.06.049.

(10) Gómez-Santacana, X.; Pittolo, S.; Rovira, X.; Lopez, M.; Zussy, C.; Dalton, J. A. R.; Faucherre, A.; Jopling, C.; Pin, J.-P.; Ciruela, F.; Goudet, C.; Giraldo, J.; Gorostiza, P.; Llebaria, A. Illuminating Phenylazopyridines To Photoswitch Metabotropic Glutamate Receptors: From the Flask to the Animals. ACS Cent. Sci. 2017, 3 (1), 81-91. https://doi.org/10.1021/acscentsci.6b00353.

(11) Hüll, K.; Fernández-Dueñas, V.; Schönberger, M.; López-Cano, M.; Trauner, D.; Ciruela, F. Optical Control of Adenosine-Mediated Pain Modulation. Bioconjug. Chem. 2021, 32 (9), 1979-1983. https://doi.org/10.1021/acs.bioconjchem.1c00387.

(12) Frank, J. A.; Antonini, M.-J.; Chiang, P.-H.; Canales, A.; Konrad, D. B.; Garwood, I. C.; Rajic, G.; Koehler, F.; Fink, Y.; Anikeeva, P. In Vivo Photopharmacology Enabled by Multifunctional Fibers.
ACS Chem. Neurosci. 2020, 11 (22), 3802-3813. https://doi.org/10.1021/acschemneuro.0c00577.

(13) Urban, P.; Pritzl, S. D.; Konrad, D. B.; Frank, J. A.; Pernpeintner, C.; Roeske, C. R.; Trauner, D.; Lohmüller, T. Light-Controlled Lipid Interaction and Membrane Organization in Photolipid Bilayer Vesicles. Langmuir 2018, 34 (44), 13368-13374. https://doi.org/10.1021/acs.langmuir.8b03241.

(14) Frank, J. A.; Moroni, M.; Moshourab, R.; Sumser, M.; Lewin, G. R.; Trauner, D. Photoswitchable Fatty Acids Enable Optical Control of TRPV1. Nat. Commun. 2015, 6, 7118. https://doi.org/10.1038/ncomms8118 https://www.nature.com/articles/ncomms8118\#supplementary-information.

(15) Küllmer, F.; Gregor, L.; Arndt, H.-D. Property-Selected Asymmetric Azobenzenes for Photoswitchable Ligands. ChemRxiv 2022. https://doi.org/10.26434/chemrxiv-2022-fd950.

(16) Müller-Deku, A.; Meiring, J. C. M.; Loy, K.; Kraus, Y.; Heise, C.; Bingham, R.; Jansen, K. I.; Qu, X.; Bartolini, F.; Kapitein, L. C.; Akhmanova, A.; Ahlfeld, J.; Trauner, D.; Thorn-Seshold, O. Photoswitchable Paclitaxel-Based Microtubule Stabilisers Allow Optical Control over the Microtubule Cytoskeleton. Nat. Commun. 2020, 11 (1), 4640. https://doi.org/10.1038/s41467-020-183896.

(17) Volgraf, M.; Gorostiza, P.; Numano, R.; Kramer, R. H.; Isacoff, E. Y.; Trauner, D. Allosteric Control of an Ionotropic Glutamate Receptor with an Optical Switch. Nat. Chem. Biol. 2006, 2 (1), 4752. https://doi.org/10.1038/nchembio756.

(18) Wegener, M.; Hansen, M. J.; Driessen, A. J. M.; Szymanski, W.; Feringa, B. L. Photocontrol of Antibacterial Activity: Shifting from UV to Red Light Activation. J. Am. Chem. Soc. 2017, 139 (49), 17979-17986. https://doi.org/10.1021/jacs.7b09281.

(19) Konrad, D. B.; Savasci, G.; Allmendinger, L.; Trauner, D.; Ochsenfeld, C.; Ali, A. M. Computational Design and Synthesis of a Deeply Red-Shifted and Bistable Azobenzene. J. Am. Chem. Soc. 2020, $\quad 142 \quad$ (14), 6538-6547. https://doi.org/10.1021/jacs.9b10430.

(20) Konrad, D. B.; Frank, J. A.; Trauner, D. Synthesis of Redshifted Azobenzene Photoswitches by Late-Stage Functionalization. Chemistry 2016, 22 (13), 4364-4368. https://doi.org/10.1002/chem.201505061.

(21) Bléger, D.; Schwarz, J.; Brouwer, A. M.; Hecht, S. O-Fluoroazobenzenes as Readily Synthesized Photoswitches Offering Nearly Quantitative Two-Way Isomerization with Visible Light. J. Am. Chem. Soc. 2012, 134 (51), 20597-20600. https://doi.org/10.1021/ja310323y.

(22) Beharry, A. A.; Sadovski, O.; Woolley, G. A. Azobenzene Photoswitching without Ultraviolet Light. J. Am. Chem. Soc. 201 1, 133 (49), 19684-19687. https://doi.org/10.1021/ja209239m.

(23) Samanta, S.; McCormick, T. M.; Schmidt, S. K.; Seferos, D. S.; Woolley, G. A. Robust Visible Light Photoswitching with OrthoThiol Substituted Azobenzenes. Chem. Commun. 2013, 49 (87), 10314-10316. https://doi.org/10.1039/C3CC46045B.

(24) Samanta, S.; Beharry, A. A.; Sadovski, O.; McCormick, T. M.; Babalhavaeji, A.; Tropepe, V.; Woolley, G. A. Photoswitching Azo Compounds in Vivo with Red Light. J. Am. Chem. Soc. 2013, 135 (26), 9777-9784. https://doi.org/10.1021/ja402220t.

(25) Forber, C. L.; Kelusky, E. C.; Bunce, N. J.; Zerner, M. C. Electronic Spectra of Cis- and Trans-Azobenzenes: Consequences of Ortho Substitution. J. Am. Chem. Soc. 1985, 107 (21), 5884-5890. https://doi.org/10.1021/ja00307a009.

(26) Bisle, H.; Rau, H. Fluorescence of Noncyclic Azo Compounds with a Low-Lying $1\left(\mathrm{n}, \Pi^{*}\right)$ State. Chem. Phys. Lett. 1975, 31 (2), 264-266. https://doi.org/10.1016/0009-2614(75)85017-2.

(27) Hansen, M. J.; Lerch, M. M.; Szymanski, W.; Feringa, B. L. Direct and Versatile Synthesis of Red-Shifted Azobenzenes. Angew. Chem. 2016, 128 (43), 13712-13716. https://doi.org/10.1002/ange.201607529.

(28) Yin, Z.; Jiang, X.; Sun, P. Palladium-Catalyzed Direct Ortho Alkoxylation of Aromatic Azo Compounds with Alcohols. J. Org. Chem. 
2013, $\quad 78 \quad$ (19), $\quad 10002-10007$. https://doi.org/10.1021/jo401623j.

Sailer, A.; Meiring, J. C. M.; Heise, C.; Pettersson, L. N.; Akhmanova, A.; Thorn-Seshold, J.; Thorn-Seshold, O. Pyrrole Hemithioindigo Antimitotics with Near-Quantitative Bidirectional Photoswitching Photocontrol Cellular Microtubule Dynamics with Single-Cell Precision. Angew. Chem. Int. Ed. 2021, $n / a$ (n/a). https://doi.org/10.1002/anie.202104794.

(30) Gao, L.; Meiring, J. C. M.; Varady, A.; Ruider, I. E.; Heise, C.; Wranik, M.; Velasco, C. D.; Taylor, J. A.; Terni, B.; Standfuss, J.; Cabernard, C. C.; Llobet, A.; Steinmetz, M. O.; Bausch, A. R.; Distel, M.; Thorn-Seshold, J.; Akhmanova, A.; Thorn-Seshold, O. In Vivo Photocontrol of Microtubule Dynamics and Integrity, Migration and Mitosis, by the Potent GFP-Imaging-Compatible Photoswitchable Reagents SBTubA4P and SBTub2M. bioRxiv 2021. https://doi.org/10.1101/2021.03.26.437160.

(31) Iwasawa, T.; Tokunaga, M.; Obora, Y.; Tsuji, Y. Homogeneous Palladium Catalyst Suppressing Pd Black Formation in Air Oxidation of Alcohols. 2.

(32) Landry, M. L.; Crawford, J. J. LogD Contributions of Substituents Commonly Used in Medicinal Chemistry. ACS Med. Chem. Lett. 2020, 11 (1), 72-76. https://doi.org/10.1021/acsmedchemlett.9b00489.

(33) Arrivault, S.; Guenther, M.; Fry, S. C.; Fuenfgeld, M. M. F. F.; Veyel, D.; Mettler-Altmann, T.; Stitt, M.; Lunn, J. E. Synthesis and
Use of Stable-Isotope-Labeled Internal Standards for Quantification of Phosphorylated Metabolites by LC-MS/MS. Anal. Chem. 2015, 87 (13), 6896-6904. https://doi.org/10.1021/acs.analchem.5b01387.

(34) Urban, P.; Pritzl, S. D.; Ober, M. F.; Dirscherl, C. F.; Pernpeintner C.; Konrad, D. B.; Frank, J. A.; Trauner, D.; Nickel, B.; Lohmueller, T. A Lipid Photoswitch Controls Fluidity in Supported Bilayer Membranes. Langmuir 2020, 36 (10), 2629-2634. https://doi.org/10.1021/acs.langmuir.9b02942.

(35) Thorn-Seshold, O. Photoswitchable Cytotoxins. In Molecular Photoswitches; Wiley, 2022.

(36) Maier, M. S.; Hüll, K.; Reynders, M.; Matsuura, B. S.; Leippe, P.; Ko, T.; Schäffer, L.; Trauner, D. Oxidative Approach Enables Efficient Access to Cyclic Azobenzenes. J. Am. Chem. Soc. 2019, 141 (43), 17295-17304. https://doi.org/10.1021/jacs.9b08794.

(37) Lentes, P.; Stadler, E.; Röhricht, F.; Brahms, A.; Gröbner, J.; Sönnichsen, F. D.; Gescheidt, G.; Herges, R. Nitrogen Bridged Diazocines: Photochromes Switching within the Near-Infrared Region with High Quantum Yields in Organic Solvents and in Water. J. Am. Chem. Soc. 2019, 141 (34), 13592-13600. https://doi.org/10.1021/jacs.9b06104.

Authors are required to submit a graphic entry for the Table of Contents (TOC) that, in conjunction with the manuscript title, should give the reader a representative idea of one of the following: A key structure, reaction, equation, concept, or theorem, etc., that is discussed in the manuscript. Consult the journal's Instructions for Authors for TOC graphic specifications.

\section{Insert Table of Contents artwork here}

